\begin{tabular}{|c|c|}
\hline Title & Topology Optimization of Synchronous Reluctance Motor Using Normalized Gaussian Network \\
\hline Author(s) & Sato, Shunpei; Sato, Takahiro; Igarashi, Hajime \\
\hline Citation & $\begin{array}{l}\text { IEEE transactions on magnetics, 51(3), } 8200904 \\
\text { https://doi.org/10.1109/T MA G.2014.2359679 }\end{array}$ \\
\hline Issue Date & 2015-03 \\
\hline Doc URL & http://hdl.handle.net/2115/59620 \\
\hline Rights & $\begin{array}{l}\text { (c) } 2015 \text { IEEE. Personal use of this material is permitted. Permission from IEEE must be obtained for all other uses, in } \\
\text { any current or future media, including reprinting/republishing this material for advertising or promotional purposes, } \\
\text { creating new collective works, for resale or redistribution to servers or lists, or reuse of any copyrighted component of } \\
\text { this work in other works. }\end{array}$ \\
\hline Tyре & article (author version) \\
\hline File Information & 70041.pdf \\
\hline
\end{tabular}

Instructions for use 


\title{
Topology Optimization of Synchronous Reluctance Motor Using Normalized Gaussian Network
}

\author{
Shunpei Sato, Takahiro Sato, Hajime Igarashi, Member, IEEE \\ Graduate School of Information Science and Technology, Hokkaido University, \\ Kita 14, Nishi 9, Kita-ku, Sapporo, JAPAN,
}

\begin{abstract}
This paper presents the topology optimization of a synchronous reluctance motor using the normalized Gaussian network. In the optimization, the average torque and iron loss are considered. In the resultant motor, the area of the rotor surface adjacent to the stator is found to be reduced when the weight for the iron loss is sufficiently large. On the other hand, large flux barriers present in the rotor when the average torque is maximized without considering the iron loss.
\end{abstract}

Index Terms - Iron loss, Normalized Gaussian network, Synchronous reluctance motor, Topology optimization

\section{INTRODUCTION}

$\mathrm{T}$ HE synchronous reluctance motor (SynRM) has widely been used due to its simple structure and low manufacturing cost. However, it is known that the maximum torque and the efficiency of SynRMs are inferior to those of permanent magnet motors [1]. To solve those drawbacks, it is important to optimize the rotor shape of SynRM aiming at increase in the torque without increasing iron losses.

To realize SynRMs with large torque, multiple flux barrier designs have been introduced, and their rotor shapes have been optimized based on parameter optimization approaches [2-4]. Although the torque has successfully been increased by these optimizations, there would exist better flux barriers which cannot be approximated by the assumed parameterized shapes.

The topology optimization allows us to obtain optimized shapes without parameterization. Because no assumptions are necessary for the topology optimization, it is advantageous especially for initial concept design. The rotor of SynRMs has been optimized using the level set method [5-6] so far which is a gradient-based topology optimization method. However, the level set method has difficulties in evaluation of iron losses which are often expressed by a non-differentiable function. On the other hand, the stochastic topology optimization method called on/off method [7] can easily treat with the nondifferentiable objective functions. There are little studies on performance of the on/off method applied to optimization of SynRMs. It is known that the on/off method tends to result in unacceptably complicated shapes when there are large number of cells to which on/off states are assigned [8].

In this work, the rotor of SynRMs is optimized using the on/off method based on the normalized Gaussian network (NGnet) [9]. The present method is shown to converge to relatively simple shapes which are suitable for industrial realization. Moreover, the iron loss is newly taken into account in the optimization. It is shown that the resultant rotor shapes drastically change when the iron loss is introduced in the objective function.

\section{OPTIMIZATION AND CALCULATION METHODS}

\section{A. Topology optimization using NGnet}

In the topology optimization of SynRM using NGnet [9], the rotor shape is determined from the output of NGnet, which is given by the weighting sum of normalized Gaussian functions $G_{i}$, as shown in Fig. 1. The output of NGnet $y(x)$ is computed from

$y(\boldsymbol{x})=\sum_{i=1}^{N} w_{i} b_{i}(\boldsymbol{x})$,
$b_{i}(\boldsymbol{x})=G_{i}(\boldsymbol{x}) / \sum_{j=1}^{N} G_{j}(\boldsymbol{x})$,

where $N$ and $w_{i}$ are the number of Gaussian function and weighting coefficient, respectively. The state $S_{e}$ of cell $e$ in the design region, which is assumed identical to a finite element in this study, is determined from the output of NGnet as follows:

$S_{e} \leftarrow \begin{cases}\text { on } & y\left(x_{\boldsymbol{e}}\right) \geq 0, \\ \text { off } & y\left(\boldsymbol{x}_{\boldsymbol{e}}\right)<0,\end{cases}$

where $\boldsymbol{x}_{\boldsymbol{e}}$ is the center of element $e$. When $S_{e}$ is on (off), its material attribute is set to steel iron (air). In the optimization, $\boldsymbol{w}=\left\{w_{i} \mid i=1,2, \ldots, N\right\}$ is determined so as to maximize the objective function by the real-coded genetic algorithm (GA) subjected to constraints. The Gaussian centers and their deviations are determined before the optimization so that they fully cover the design region.

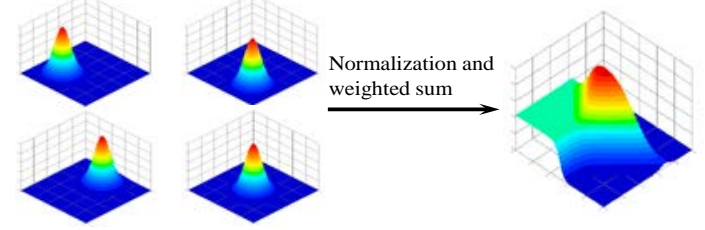

Fig. 1. The output of NGnet in case of four Gaussian functions.

\section{B. Iron loss computation}

To evaluate the iron loss, we employ the 1-D FE method [10] in which magnetic saturation and eddy current are taken 
into account. It has been shown that this method can analyze the iron loss of the rotating machineries at satisfactory accuracy.

\section{1) Computation of eddy current loss}

In the 1-D FE method [10], the eddy current in the electrical steel sheet is computed in the post processing of magnetostatic FE analysis. As shown in Fig. 2, we analyze the field in the half region of the electrical steel sheet because of the symmetry. In this method, we solve the following equations:

$\frac{\partial}{\partial z}\left(v \frac{\partial A_{x}}{\partial z}\right)=\sigma \frac{\partial A_{x}}{\partial t}$

$\frac{\partial}{\partial z}\left(v \frac{\partial A_{y}}{\partial z}\right)=\sigma \frac{\partial A_{y}}{\partial t}$

under the boundary conditions given by

$$
\begin{aligned}
& \left.A_{x}\right|_{z=d / 2}=\frac{B_{y} d}{2}, \\
& \left.A_{y}\right|_{z=d / 2}=-\frac{B_{x} d}{2}, \\
& \left.A_{x}\right|_{z=0}=\left.A_{y}\right|_{z=0}=0,
\end{aligned}
$$

where $A_{x}, A_{y}, v, \sigma$ and $d$ are $x$ and $y$ components of vector potential, magnetic reluctivity, conductivity and thickness of the electrical steel sheet, respectively. From this method, the eddy current in one electrical steel sheet can be computed considering the skin effect. The eddy current loss $W_{e}$ is then computed from

$$
W_{e}=\sum_{i=1}^{N_{E}} \frac{2 \Delta V_{i}}{T d} \int_{0}^{T} \int_{0}^{\frac{d}{2}} \kappa \sigma\left|\frac{\partial \boldsymbol{A}}{\partial t}\right|^{2} d z d t,
$$

where $N_{E}, \Delta V_{i}, T$ and $\kappa$ are the number of elements in the design region, the volume of the $i$-th element, the period and the correction coefficient for excess eddy current loss, respectively.

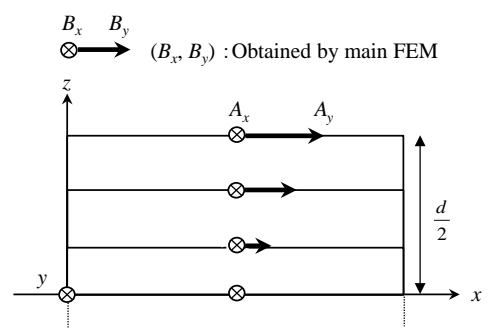

Fig. 2. 1-D FE model of electrical steel sheet.

\section{2) Computation of hysteresis loss}

The hysteresis loss is also computed in the post processing using the 1-D FE analysis [10]. In this method, the hysteresis loss is computed from

$$
W_{h}=\frac{K_{h} D}{T} \sum_{i=1}^{N_{E}} \sum_{k=1}^{n e} \frac{V^{i}}{2}\left(\sum_{j=1}^{m_{x i}}\left(B_{x}^{i j}\right)^{2}+\sum_{j=1}^{m_{y i}}\left(B^{i j}\right)^{2}\right),
$$

where $K_{h}, D$ and ne are the coefficient of hysteresis loss, the density of electrical steel iron and the number of 1-D FE, $m_{x}$ and $m_{y}$ are the number of extrema in the $x$ and $y$ component of magnetic flux density, respectively. $B^{i j}{ }_{x}$ and $B^{i j}{ }_{y}$ represent the amplitudes of the magnetic induction determined from the 1-D FE analysis.

\section{OPTIMIZATIONS OF SYNRM}

\section{A. Optimization problems}

Fig. 3 shows a simple salient pole motor (reference model) whose performance will be compared with that of the motors optimized using the present method. The shape paremeters, height and angle of the salient poles, in the reference model are optimized so that the torque is maximized. The average torque $T_{0}$ and the iron loss $W_{0}$ of this model are described in Fig.3. The stator structure of this model is based on the IEEJ D-model [7, 11] whose specifications are summarized in Table I. In the FE analysis, the nonlinear magnetic property of the core, which is assumed to be made of 50A470, is considered. The material parameters of 50A470 for loss computation are summarized in Table II [12].

The design region in which the rotor shape is determined by the present method is shown in Fig. 4. The number of finite elements in the design region is 2256. For the NGnet-based topology optimization method, 86 Gaussians whose deviation is $10^{-3}$ are uniformly deployed in the design region. The chromosome size in GA is 86. Because of large chromosome size in GA, 800 individuals are created for the first generation, and 100 children are generated in every generation. The optimization is performed over 50 generations. The total function calls for FE analysis are $800+100 \times 50$.

Because our main aim is to maximize the average torque suppressing the increase in iron loss, the torque ripple is not taken into account in the optimization.

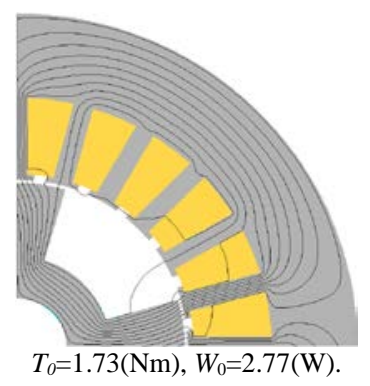

Fig. 3. Reference model

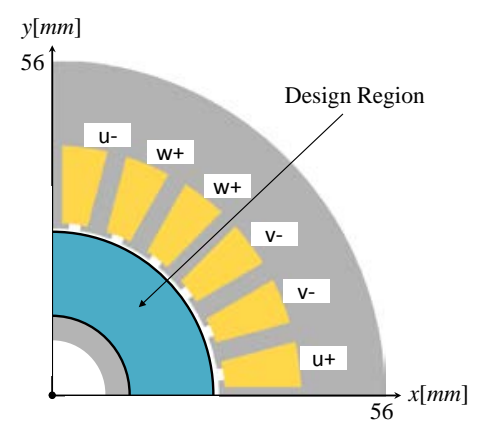

Fig. 4. SynRM for optimization based on IEEJ D-model $[7,11]$

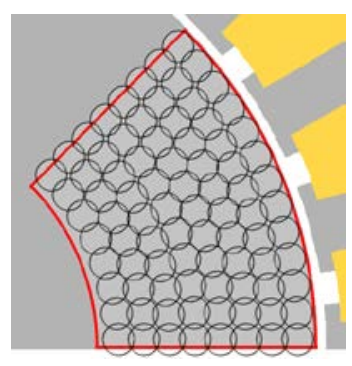

Fig. 5. Distribution of Gaussian functions 
TABLE I

SPECIFICATIONS OF THE REFERENCE MOTOR

\begin{tabular}{cc}
\hline \hline Phase and pole & 3-phase, 4-poles \\
Phase current (Arms) & 6 \\
Rotation speed (rpm) & 1500 \\
Current phase angle (degree) & 45 \\
Stator and rotor core grade & $50 \mathrm{~A} 470$ \\
\hline \hline
\end{tabular}

TABLE II

CHARACTERISTICS OF ELECTRICAL STEEL SHEET (50A470)

\begin{tabular}{cc}
\hline \hline$K_{h}$ & $2.71 \times 10^{-2}$ \\
$D\left(\mathrm{~kg} / \mathrm{m}^{3}\right)$ & $7.70 \times 10^{3}$ \\
$\kappa$ & 0.96 \\
Conductivity $\sigma(\mathrm{S} / \mathrm{m})$ & $2.56 \times 10^{6}$ \\
Thickness $d(\mathrm{~mm})$ & 0.5 \\
\hline \hline
\end{tabular}

\section{B. Maximization of torque}

The optimization is performed only considering the average torque. The optimization problem is defined by

$$
F_{1}=\frac{T_{\text {ave }}}{T_{0}} \rightarrow \text { max., }
$$

where $T_{\text {ave }}$ and $T_{0}$ denote the average torques of the optimization model in Fig.5 and the reference model.

The optimization result is shown in Fig. 6(a). It can be found that avegrage torque is increased by 43.4 percent in comparison with that of the reference model, whereas iron loss is also increased by 50.9 percent. This result suggests that there is a trade-off relation between average toque and iron loss. The thin bridge between the flux barriers would be made thicker to increase mechanical strength for its production.

Fig. 6(b) shows the iron loss density distribution. It can be observed from this result that iron loss density in the rotor core concentrates near the rotor surface. This is due to the fact that the magnetic fields near the rotor surface have large time variations, whereas they have little changes in the region far from the surface, as pointed out in [13].

\section{Minimization of iron loss with torque constraint}

We optimize the rotor shape to minimize the iron loss keeping the average torque as large as possible. Thus, the optimization problem is defined as follows:

$$
F_{2}=-\frac{W_{\text {loss }}}{W_{0}} \rightarrow \text { max., sub. to } T_{\text {ave }}>T_{0},
$$

where $W_{\text {loss }}$ and $W_{0}$ denote the iron losses of the optimization model and the reference model. In this optimization, the penalty is imposed when the constraint is violated.

The optimization result is shown in Fig. 7(a). It is found that the iron loss is reduced by 33.9 percent in comparison with that of the reference model. Moreover, the average torque is kept almost to $T_{0}$. The resultant rotor shape shown in Fig. 7(a) is quite different from that for problem (11); the rotor surface adjacent to the stator is reduced and there are no flux barriers in the former. We can see in Fig. 7(b) that the lossy region near the stator becomes small.

\section{Maximization of torque with iron loss constraint}

Finally, we perform the optimization to maximize the average torque under the condition that the iron loss is lower than that of the reffecence model. The optimization problem is defined as follows:

$F_{3}=\frac{T_{\text {ave }}}{T_{0}} \rightarrow$ max., sub. to $W_{\text {loss }}<W_{0}$.

The constraint is again included in the object function as a penalty term.

The optimization result is shown in Fig. 8(a). The avegrage torque is now increased by approximately 8.7 percent in comparison with that of the reference model, while the iron loss is kept almost the same as that of the reference model.

The width of the resultant salient poles in Fig. 8(a) is found to be larger than that in Fig. 7(a). On the other hand, the air gap in the middle of the salient poles is wider in the former.

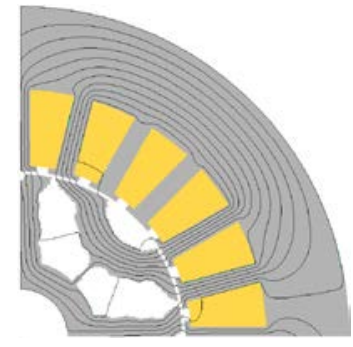

$T_{\text {ave }}=2.48(\mathrm{Nm}), W_{\text {loss }}=4.18(\mathrm{~W})$. (a) Optimized shape

Fig. 6. Optimization result to problem (11)

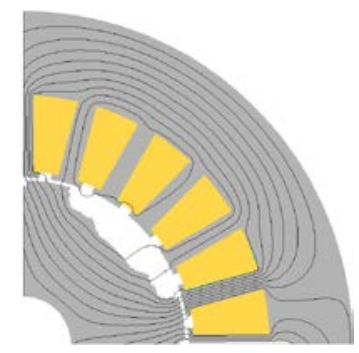

$T_{\text {ave }}=1.73(\mathrm{Nm}), W_{\text {loss }}=1.83(\mathrm{~W})$. (a) Optimized shape

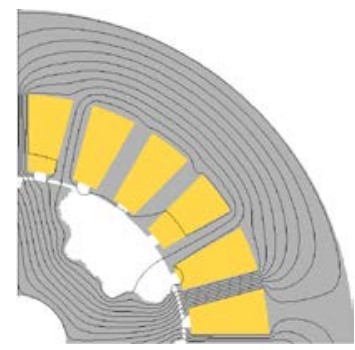

$T_{\text {ave }}=1.88(\mathrm{Nm}), W_{\text {loss }}=2.70(\mathrm{~W})$. (a) Optimized shape

Fig. 8. Optimization result to problem (13) Fig. 7. Optimization result to problem (12)

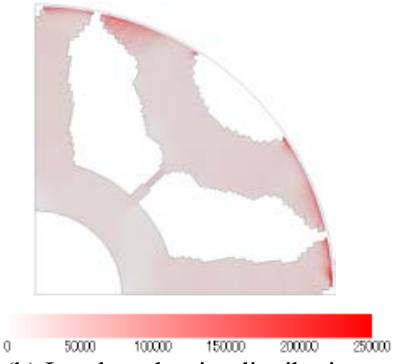

(b) Iron loss density distribution $\left(\mathrm{W} / \mathrm{m}^{3}\right)$

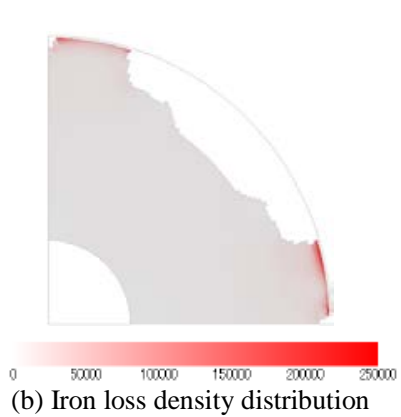

$\left(\mathrm{W} / \mathrm{m}^{3}\right)$ (b) Iron loss density distribution

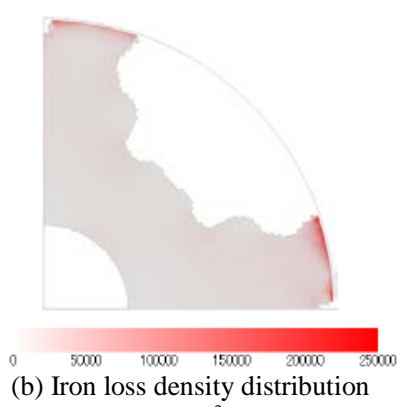

$\left(\mathrm{W} / \mathrm{m}^{3}\right)$ 


\section{E. Discussions}

Although the optimized shapes shown in Figs. 6, 7 and 8 seem relatively simple, their rotor surfaces are wavy. Their shapes should be simplified for productions. To consider the effect of the shape simplification on the performance, the rotor shape in Fig. 6 is simplified and the performance is evaluated. The results are shown in Fig. 9(a), from which we can see that the simplification gives no significant effects on the performance. In addition, the mechanical strength of the revised rotor is evaluated by the mechanical FEM. The motor speed is assumed to be 15000rpm which is ten times as large as the rated speed. The distribution of stress in the rotor core is shown in Fig. 9(b). It can be seen from Fig. 9(b) that the maximum stress $S_{\max }$ is $88.17 \mathrm{MPa}$, which is sufficiently smaller than the yield stress of 50A470.

Furthermore, the shape expression capability of present method is considered because the optimized results shown in Figs. 7-8 are relatively simple compared to the rotor shapes mentioned in [3-4] which have multiple flux barriers. This might be due to the fact that the number of Gaussians is insufficient to express multiple flux barriers. For this reason, to improve the shape expression capability, the optimization is performed using 125 Gaussians in which the optimization problem (11) is solved. As a result, we find that the resultant shape is almost identical to that in Fig. 6(a). This suggests that the shape expression capability is sufficient for at least problem (11). The rotor shapes of the best individuals during the optimization process are shown in Fig. 10. It is found from Fig. 10 that the rotor shapes are much different from that in Fig. 6(a) although they will evolve to the simple rotor shape shown in Fig. 6(a).

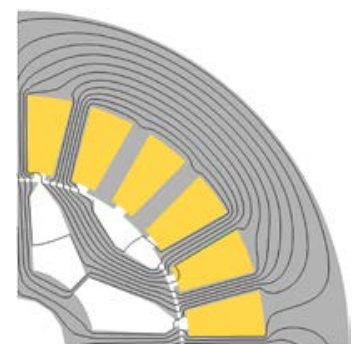

$T_{a v e}=2.39(\mathrm{Nm}), W_{\text {loss }}=3.40(\mathrm{~W})$. (a) Simplified shape

Fig. 9. Simplification of motor in Fig.6 for production

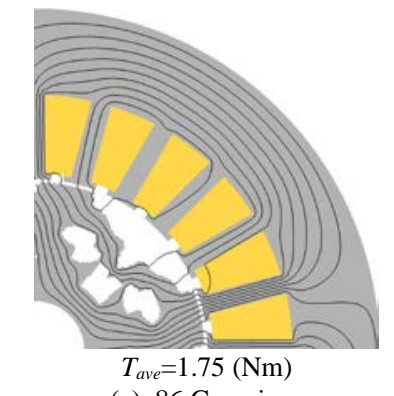

- (a) 86 Gaussians
Fig. 10. Rotor shape of best individuals at 15th generation of GA process

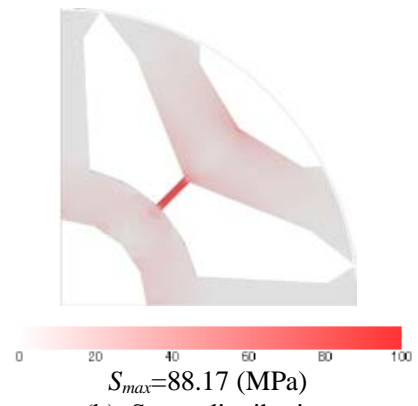

(b) Stress distribution

\section{CONCLUSIONS}

In this paper, the topology optimizations of SynRMs considering both torque and iron loss have been performed using the present method. It has been shown that the optimized shapes drastically change depending on the objectives. It has been suggested that there would be trade-off relation between average torque and iron-loss. The torque can be increased by the flux barriers and wide rotor surface adjacent to the stator, while the latter should be made smaller to reduce the iron loss.

\section{REFERENCES}

[1] H. Murakami, Y. Honda, H. Kiriyama, S. Morimoto, and Y. Takeda, "The performance comparison of SPMSM, IPMSM, and SynRM in use as air-conditioning compressor," in Conf. Rec. IEEE-IAS Annu. Meeting, pp. 840-845, 1999.

[2] T. Matsuo and T. A.Lipo, "Rotor Design Optimization of Synchronous Reluctance Machine,” IEEE Trans. Energy Conversion, vol.9, No.2, 1994.

[3] F. Cupertino, G. Pellegrino, and C. Gerada, "Design of synchronous reluctance machines with multi-objective optimization algorithms," Energy Conversion Congress and Exposition (ECCE), 2013 IEEE International, pp. 1858-1865, 2013.

[4] G. Pellegrino, F. Cupertino, and C. Gerada, "Barriers shapes and minimum set of rotor parameters in the automated design of Synchronous Reluctance machines," Electric Machines and Drives Conference (IEMDC), 2013 IEEE International, pp. 1204-1210, 2013.

[5] Y. S. Kim and I. H. Park, "Topology optimization of rotor in synchronous reluctance motor using level set method and shape design sensitivity," IEEE Trans. Applied Superconductivity, vol. 20, no. 3, pp. 1093-1096, 2010.

[6] S. H. Heo, M. K. Baek, K. H. Lee, S. G. Hong, and I. H. Park, "Shape and topology optimization of rotor in synchronous reluctance motor using continuum sensitivity and adaptive level set method," ICEMS, pp. 129-133, 2013.

[7] N. Takahashi, T. Yamada, S. Shimose, and D. Miyagi, "Optimization of rotor of actual IPM motor using ON/OFF method," IEEE Trans. Magnetics, vol. 47, no. 5, pp. 1262-1265, 2011.

[8] T. Sato, K. Watanabe, and H. Igarashi, "A modified immune algorithm with spatial filtering for multiobjective topology optimisation of electromagnetic devices,” COMPEL, vol. 33, Iss: 3, pp. 821- 833, 2014.

[9] T. Sato, K. Watanabe, and H. Igarashi, "A Topology Optimization Method for Electrical Machines Based on Normalized Gaussian Network," IEEJ Joint Tech. Meeting on Static Apparatus and Rotating Machinery, pp.17-22, 2013.

[10] K. Yamazaki and N. Fukushima, "Torque and Loss Calculation of Rotating machines Considering Laminated Cores Using Post 1-D Analysis,” IEEE Trans. Magnetics, vol. 47, No. 5, 2011.

[11] Technical report of the institute of electrical engineering of Japan, industry application society, No.776, 2000.

[12] K. Yamazaki, A. Suzuki, M. Ohto, and T. Takakura, "Harmonic Loss and Torque Analysis of High-Speed Induction Motors," IEEE Trans. Industry Application, vol. 48, No. 3, 2012.

[13] K. Yamazaki and N. Fukushima, "Iron Loss Model for Rotating Machines Using Direct Eddy Current Analysis in Electrical Steel Sheets," IEEE Trans. Energy Conversion, vol. 25, No. 3, 2010. 